\title{
Pulmonary Edema: A Unifying Pathophysiological Formula
}

\section{Vladimir Tilman*}

Sheba Medical Center at Tel Hashomer, Holon, Israel

*Corresponding author: Vladimir Tilman, Sheba Medical Center at Tel Hashomer, Rehabilitation Hospital, Eilat 33 apt 4, Holon, 58370, Israel, Tel: 972-0547345525; Email:water5945@gmail.com

Received date: February 9, 2015; Accepted date: March 30, 2015; Published date: April 2, 2015

Copyright: $\odot 2015$ Tilman V. This is an open-access article distributed under the terms of the Creative Commons Attribution License, which permits unrestricted use, distribution, and reproduction in any medium, provided the original author and source are credited.

\begin{abstract}
Pulmonary edema is a significant complication of a number of disease and conditions. Its main manifestation is the leakage of fluid from the pulmonary capillaries into the cavity of the alveoli through the alveolar-capillary membrane. This leads to a number of severe clinical manifestations and requires immediate treatment. The underlying mechanisms causing this complication include cardiogenic and noncardiogenic factors. A formula is proposed to attempt to unify the causes of pulmonary edema, reflecting the relationship of all factors involved in the process. The formula is simple and evident, and covers all the factors that cause or contribute to the development of pulmonary edema. It also explains the pathogenesis of cardiogenic and noncardiogenic pulmonary edema and their relationship.
\end{abstract}

Keywords: Heart failure; pulmonary edema; Alveolarcapillary membrana

\section{Introduction}

Pulmonary edema (PE) is a common cause of morbidity and mortality in the critically ill. PE is caused by transudation of fluid into the pulmonary interstitium and alveoli, and may have cardiac or noncardiac etiology. Physiologically, exchange of fluid normally occurs between the vascular bed and the interstitium of the alveolarcapillary membrane. PE occurs when the net flux of fluid from the vasculature into the interstitial space is increased. The Starling relationship determines the fluid balance between the alveoli and the vascular bed. Net flow of fluid across a membrane is determined by applying the following equation:

$$
\mathrm{Q}=\mathrm{K} \text { (P1cap - P1is) - I (P2cap - P2is), }
$$

where $\mathrm{Q}$ is net fluid filtration; $\mathrm{K}$ is a constant called the filtration coefficient; P1cap is capillary hydrostatic pressure, which tends to force fluid out of the capillary; P1is is hydrostatic pressure in the interstitial fluid, which tends to force fluid into the capillary; I is the reflection coefficient, which indicates the effectiveness of the capillary wall in preventing protein filtration; P2cap is the colloid osmotic pressure of plasma, which tends to pull fluid into the capillary, and P2is is the colloid osmotic pressure in the interstitial fluid, which pull fluid out of the capillary [1]. The Starling equation is helpful, especially to conceptualize the role of increased capillary hydrostatic pressure, but is not very useful clinically, only in specialized scientific laboratories, since it is not possible to measure all six of the unknown values, and it is not directed towards other causes of the development of PE.

\section{Methods}

I am proposing a very clear and demonstrative formula of PE that includes all pathophysiological mechanisms, and will help in understanding the development of both cardiogenic and noncardiogenic PE. I want to underline that the proposed formula has a theoretical value; it is a pathophysiological, functional formula, which does not need mathematical measurements.

Among the forces responsible for fluid transference to and from the interstitium are:

- Pulmonary capillary hydrostatic pressure, under the action of which fluid tends to leak through the alveolar-capillary membrane;

- Colloid-osmotic pressure of plasma that keeps the fluid in the capillary bed;

- The state of the alveolar-capillary membrane;

- The state of the pulmonary lymphatic system which drains the excess fluid from the interstitial space of the alveolar-capillary membrane;

- The level of atmosphere pressure in the alveoli.

In normal conditions the relationship of the above-mentioned factors can be presented as a simple formula:

$\mathrm{PCHP} \leq \sum(\mathrm{COP}+\mathrm{M}+\mathrm{L}+\mathrm{At})$

Where, PCHP - pulmonary capillary hydrostatic pressure, on value it is equal to pulmonary wedge pressure (when the mitral valve is still open at the end of diastole, the pressures in the left ventricle, left atrium, pulmonary veins and alveolar capillaries are equal) normally 8-12 $\mathrm{mm} \mathrm{Hg}$.

$\leq$ - mathematical symbol "no more than", it means that the left part of formula should not exceed the right part in normal conditions.

$\Sigma$ - functional sum of factors of the right part of the formulaindicating a synergism of actions individually or in combinations, against the factors of the left part of the formula.

COP - colloid osmotic pressure of plasma, which includes osmotic pressure of electrolytes and oncotic pressure of proteins and their complexes. Normal COP of plasma of blood $(25 \mathrm{~mm} \mathrm{Hg})$ is higher than pulmonary capillary hydrostatic pressure $(8-12 \mathrm{~mm} \mathrm{Hg})$ and exerts a powerful drive to keep the interstitium and alveoli dry. Proteins, especially albumin are the main contributors to the COP. The normal level of albumin is $4-5 \mathrm{~g} / \mathrm{dl}$, with each $1 \mathrm{~g}$ albumin creating 
4-5 $\mathrm{mm} \mathrm{Hg}$ of oncotic pressure. Thus, the oncotic pressure is strongly dependent on the level of albumin in the blood [2]. Interestingly, the pulmonary capillaries appear to have a greater permeability to albumin, causing higher interstitial oncotic pressure $(\sim 18 \mathrm{~mm} \mathrm{Hg})$. Thus, while hypoalbuminemia as an independent variable can lead to peripheral edema by lowering the transcapillary oncotic pressure gradient, it does not generally produce pulmonary edema, as it also lowers the interstitial oncotic pressure.

M - Status of the alveolar-capillary membrane. Pulmonary capillary blood and alveolar gas are separated by the alveolar-capillary membrane, which consists of 3 anatomically different layers: 1) the capillary endothelium; 2) the interstitial space, which may contain tissue, fibroblasts, and macrophages; and 3) the alveolar epithelium with surfactant.

L - Status of the lymphatic system. The lymphatic drainage plays an important role in maintaining an adequate fluid balance in the lung by removing solutes, colloids, and liquid from the interstitial space at a rate of approximately $10-20 \mathrm{ml} / \mathrm{h}$ and therefore participates in prevention of pulmonary congestion. In normal circumstances the interstitial space can increase its volume by as much as $40 \%$ without resulting in $\mathrm{PE}$.

At - the level of the atmospheric barometric pressure in alveoli. The pressure of gas in the alveolar space counteracts the PCHP and the exit of the fluid through the alveolar-capillary membrane.

Thus in normal state there is a dynamic balance between factors on the left side of the formula, that promote the exit of the liquid from the pulmonary capillaries through the alveolar membrane and factors on the right side of the formula, counteracting to this process, reflected in the formula by the sign " no more than" $(\leq)$.

Respectively, the change of the balance in favor of the left part of the formula and the change of the sign from $(\leq)$ - "no more" to the sign (>)-" more" means fluid leaks from the pulmonary capillaries through the alveolar membrane into alveoli with development of PE. Thus the formula for pulmonary edema:

$\mathrm{PCHP}>\sum(\mathrm{COP}+\mathrm{M}+\mathrm{L}+\mathrm{At})$

It should be noted that changes of the sign from "no more than" $(\leq)$ to $(>)$ the " more than " can be either due to "increasing" the factor that influences the left side of the formula, or " reducing " the influence of the factors of the right side of the formula. Increasing PCHP means the development of PE because of the cardiovascular causes, i.e. it is cardiogenic PE. Reducing the influence of $\sum$ (a single factor or their combination) also leads to development of $\mathrm{PE}$, but it is noncardiogenic PE (Table 1).

\begin{tabular}{|l|l|l|}
\hline Causes of cardiogenic PE & & causes of noncardiogenic PE \\
\hline PCHP & $>$ & $\sum(C O P+M+L+A t)$ \\
\hline
\end{tabular}

Table 1: Effect of change of sign $(\leq)$ to $(>)$

It should be noted that in clinical practice there is often a combination of factors that may contribute to development of PE or affect its course. For example, excess fluid transfusion, renal failure with inadequate fluid elimination, or travel to a high altitude (noncardiogenic factors) in an individual with compensated heart failure (cardiogenic factor) can provoke decompensation with the development of PE.
Thus the formula reflects the entire spectrum of the pathophysiological causes and cleanly demonstrates the pathogenesis of both forms of PE and their relationship.

Having the complete formula of the pulmonary edema it is now possible to consider various scenarios of the development of different forms of PE.

\section{Cardiogenic PE}

It is defined as PE due to increasing of the pulmonary capillary hydrostatic pressure that must rise to a level higher than the functional sum of the right side's factors, i.e. sign $(\leq)$ changes to sign (>).

$$
\mathrm{PCHP}>\sum(\mathrm{COP}+\mathrm{M}+\mathrm{L}+\mathrm{At})
$$

Many cardiovascular diseases (coronary artery disease, cardiomyopathy, heart valve problems, hypertension etc.) may lead to development of PE, but the main cause in all cases is increasing end diastolic pressure, pulmonary wedge pressure, and pulmonary capillary hydrostatic pressure respectively to $20 \mathrm{~mm} \mathrm{Hg}$, or more, and is a main manifestation of the heart failure. As a result of increasing PCHP, fluid is pushed through the alveolar-capillary membrane into the air sacs with development of pulmonary edema $[3,4]$.

\section{Noncardiogenic pulmonary edema}

- Reducing of colloid-osmotic pressure

$$
\mathrm{PCHP}>\sum(\mathrm{COP}+\mathrm{M}+\mathrm{L}+\mathrm{At})
$$

Significant reduction of colloid-osmotic pressure can change the ratio of the pressure in favor towards PCHP, resulting in the conditions for PE, i.e. also changing the sign $(\leq)$ to $(>)$. In clinical practice it may be due to excess amount of liquid with considerable dilution of the blood, such as caused by transfusion of hypotonic or hypooncotic solutions, dilution of the blood following freshwater drowning due to freshwater entering the alveoli, or by significant hypoalbuminemia as a serious aggravating factor towards the development of PE $[5,6]$.

- Damage of the alveolar-capillary membrane and violation of its barrier function can also promotes a fluid exit through the membrane despite maintained pulmonary capillary hydrostatic pressure, thus changing the sign $(\leq)$ to $(>)$.

$$
\mathrm{PCHP}>\sum(\mathrm{COP}+\mathrm{M}+\mathrm{L}+\mathrm{At})
$$

There are many causes for damage of the membrane inflammation, end stage of severe pneumonia, ARDS, injury of lungs, toxic effect of chemicals, such as gasoline, kerosene, various household and military organophosphate poisons, drug overdose, adverse drug reaction (among others - heroin, cocaine to aspirin, chemotherapy drugs, etc), smoke inhalation and so on [7-10].

- Lymphatic drainage incompetence can promote development of PE.

$$
\mathrm{PCHP}>\sum(\mathrm{COP}+\mathrm{M}+\mathrm{L}+\mathrm{At})
$$

As the volume excess initially enters the interstitium it is taken up by lymphatic system to be returned to the vascular system. In cases of lymphatic drainage insufficiency the fluid flow rate is inadequate to prevent accumulation in the interstitial space. The final step occurs as the fluid volume overwhelms the hydrostatic forces and the excess fluid moves into the alveoli, leading to alveolar flooding. This can also change the sign $(\leq)$ to $(>)$ creating conditions for hypostasis of the 
Citation: Tilman V (2015) Pulmonary Edema: A Unifying Pathophysiological Formula. J Cardiovasc Dis Diagn 3: 194. doi:

Page 3 of 3

lungs. Lymphatic drainage failure as an independent cause of PE is very rare (mediastinal carcinomatosis, silicosis, filariasis), but it can be an aggravating factor in combination with other factors [5,11].

- Decrease in the level of atmosphere pressure in alveoli can also shift an imbalance of pressure in favor of the relative increase of PCHP, and change the sign $(\leq)$ to $(>)$, thus creating the preconditions for the development of $\mathrm{PE}$.

$\mathrm{PCHP}>\sum(\mathrm{COP}+\mathrm{M}+\mathrm{L}+\mathrm{At})$

A typical example is high altitude pulmonary edema (HAPE), which occurs when people are in rarefied atmosphere, i.e. with reduced alveoli air pressure. HAPE has a rapid onset in healthy people who venture to elevation above 8200 feet. Similar conditions that lead to HAPE may occur with depressurization of an aircraft or spacecraft. In the literature, the description of the pathophysiology of HAPE emphasizes the reduction of partial oxygen pressure with the development of hypoxia and its effect on the body. However, the effect of overall atmospheric barometric pressure as the sum of partial pressures of the air gases (by Dalton's law) is not discussed enough. The atmospheric barometric pressure is equal to the pressure of gases in the cavity of the alveoli that confronts the PCHP. Thus a decrease atmospheric barometric pressure on the alveolar side of the alveolarcapillary membrane results in a shift in favor of a relative increase in PCHP, changing the sign $(\leq)$ to $(>)$ in the formula, i.e. the conditions for PE. Conversely, when the barometric pressure increases in the cavity of alveoli, the reverse process occurs. Examples of the significance of an increase of barometric pressure in the alveoli include during work at a depth under water, other gases such as nitrogen pass through the alveolar-capillary membrane and dissolve. Return to normal atmospheric pressure requires a gradual decompression to prevent gas embolism and decompression sickness. Another example in clinical practice in the treatment of $\mathrm{PE}$ is high pressure breathing which increases barometric pressure in the alveoli, driving fluid into the capillary bed. The use of continuous positive airway pressure BIPAP/NIPPV devices in cases of PE has been demonstrated to reduce the need of mechanical ventilation and may reduce mortality $[12,13]$.

\section{Conclusion}

This proposed formula of pulmonary edema simplifies the understanding of the pathophysiology and helps categorize the process of the development of PE. It describes most of the factors involved in the occurrence of $\mathrm{PE}$, explains the pathogenesis of the development of both cardiogenic and non-cardiogenic pulmonary edema and their relationship. I want to reiterate that the proposed formula has a theoretical value, it is a pathophysiological, functional formula, which does not need mathematical measurements. This formula can be a useful tool in studying of the pathophysiology of PE.

\section{References}

1. Berne R, Levy M (1992) Cardiovascular Physiology, 1st edn. St.Louis: Mosby Year Book, Inc.

2. Arthur G, John H (2006) "Chapter 16: The Microcirculation and the Lymphatic System". In Gruliow, Rebecca. Textbook of Medical Physiology (Book) (11th ed.). Philadelphia, Pennsylvania: Elsevier Inc. pp. 187-188.

3. Ware LB, Matthay MA (2005) Clinical practice. Acute pulmonary edema. N Engl J Med 353: 2788-2796.

4. West JB (2012) Respiratory physiology: The essentials, Lippincott WIlliams \& Wilkins, 2012.

5. Taylor AE (1981) Capillary fluid filtration. Starling forces and lymph flow. Circ Res 49: 557-575.

6. Arques S, Ambrosi P, Gelisse R, Luccioni R, Habib G (2003) Hypoalbuminemia in elderly patients with acute diastolic heart failure. J Am Coll Cardiol 42: 712-716.

7. Ware LB, Matthay MA (2000) The acute respiratory distress syndrome. N Engl J Med 342: 1334-1349.

8. Cheng IW, Matthay MA (2003) Acute lung injury and the acute respiratory distress syndrome. Crit Care Clin 19: 693-712.

9. Sporer KA, Dorn E (2001) Heroin-related noncardiogenic pulmonary edema : a case series. Chest 120: 1628-1632.

10. Heffner JE, Sahn SA (1981) Salicylate-induced pulmonary edema. Clinical features and prognosis. Ann Intern Med 95: 405-409.

11. Miserocchi G, Negrini D, Passi A, De Luca G (2001) Development of lung edema: interstitial fluid dynamics and molecular structure. News Physiol Sci 16: 66-71.

12. Hackett P, Rennie D (2002) High-altitude pulmonary edema. JAMA 287: 2275- 2278.

13. Mehta S, Jay GD, Wollard RH, Hipona RA, Connolly EM, et al. (1997) Randomized, prospective trial of bilevel versus continuous positive airway pressure in acute pulmonary edema. Critical Care Medicine 25: 620-628. 